\title{
Association of Myoinositol Transporters with Schizophrenia and Bipolar Disorder: Evidence from Human and Animal Studies
}

\author{
Marquis P. Vawter ${ }^{\mathrm{a}} \quad$ Abdul Rezzak Hamzeh ${ }^{\mathrm{b}}$ Edgar Muradyan ${ }^{\mathrm{C}}$ \\ Olivier Civellic-e Geoffrey W. Abbott ${ }^{f}$ Amal Alachkar ${ }^{c, d}$
}

\begin{abstract}
${ }^{a}$ Department of Psychiatry and Human Behavior, School of Medicine, University of California, Irvine, Irvine, CA, USA;
b John Curtin School of Medical Research, Australian National University, Canberra, ACT, Australia; 'Department of Pharmacology, School of Medicine, University of California, Irvine, Irvine, CA, USA; ${ }^{d}$ Department of Pharmaceutical Sciences, School of Medicine, University of California, Irvine, Irvine, CA, USA; ${ }^{e}$ Department of Developmental and Cell Biology, School of Medicine, University of California, Irvine, Irvine, CA, USA; ${ }^{f}$ Bioelectricity Laboratory, Department of Physiology and Biophysics, School of Medicine, University of California, Irvine, Irvine, CA, USA
\end{abstract}

\section{Keywords}

Myoinositol transporters - Human brain · SIC5a3 knockout mice $\cdot$ Schizophrenia $\cdot$ Bipolar disorder

\begin{abstract}
Evidence from animal and human studies has linked myoinositol (MI) with the pathophysiology and/or treatment of psychiatric disorders such as schizophrenia and bipolar disorder. However, there is still controversy surrounding the definitive role of $\mathrm{Ml}$ in these disorders. Given that brain $\mathrm{Ml}$ is differentially regulated by three transporters - SMIT1, SMIT2 and/or HMIT (encoded by the genes: SLC5A3, SLC5A11, and $S L C 2 A 13$, respectively) - we used available datasets to describe the distribution in mouse and human brain of the different Ml transporters and to examine changes in mRNA expression of these transporters in patients with schizophrenia and bipolar disorder. We found a differential distribution of the mRNA of each of the three MI transporters in both human and mouse brain regions. Interestingly, while individual neurons express SMIT1 and HMIT, non-neuronal cells express SMIT2, thus partially accounting for different uptake
\end{abstract}

\section{KARGER}

(c) 2019 S. Karger AG, Basel

E-Mail karger@karger.com

www.karger.com/mnp levels of $\mathrm{Ml}$ and concordance to downstream second messenger signaling pathways. We also found that the expression of MI transporters is significantly changed in schizophrenia and bipolar disorder in a diagnostic-, brain regionand subtype-specific manner. We then examined the effects of germline deletion in mice of S/C5a3 on behavioral phenotypes related to schizophrenia and bipolar disorder. This gene deletion produces behavioral deficits that mirror some specific symptoms of schizophrenia and bipolar disorder. Finally, chronic administration of MI was able to reverse particular, but not all, behavioral deficits in S/c5a3 knockout mice; MI itself induced some behavioral deficits. Our data support a strong correlation between the expression of MI transporters and schizophrenia and bipolar disorder, and suggest that brain region-specific aberration of one or more of these transporters determines the partial behavioral phenotypes and/or symptomatic pattern of these disorders.

Dr. Geoffrey W. Abbott

Department of Physiology and Biophysics, School of Medicine

University of California, Irvine

Irvine, CA 92697 (USA)

E-Mail abbottg@uci.edu

Dr. Amal Alachkar

Department of Pharmaceutical Sciences

University of California, Irvine

Irvine, CA 92697 (USA)

E-Mail aalachka@uci.edu 


\section{Introduction}

Myo-inositol (MI) is a key metabolic precursor in the phosphatidylinositol second messenger cycle, a critical component of several neurotransmitter systems including noradrenalin, serotonin, glutamate, and acetylcholine. It also acts as an osmolyte and is released from and taken up by astrocytes in response to hypotonic and hypertonic surroundings, respectively [1-3]. MI, an optically inactive isomer of inositol, is obtained from dietary sources and can also be synthesized from glucose in many human tissues.

The concentration of MI in the human brain is estimated to be $6 \mathrm{mM}$, the majority of which is synthesized in the brain, with the rest coming from peripherally synthesized MI and dietary sources. The brain levels of MI are regulated by three active transporters, sodium-dependent MI transporter-1 (SMIT1, encoded by SLC5A3), sodiumdependent MI transporter-2 (SMIT2, SLC5A11), and $\mathrm{H}^{+}$dependent MI transporter (HMIT, SLC2A13), which import $\mathrm{MI}$ across the plasma membrane by co-transporting two sodium ions (or protons) per MI molecule down the sodium concentration gradient.

MI has been linked with the pathophysiology and/or the treatment of several neuropsychiatric disorders, including depression $[4,5]$, obsessive compulsive disorder (OCD) $[6,7]$, panic disorder $[8,9]$, attention deficit hyperactivity disorder (ADHD) [10], Alzheimer's disease $[11,12]$, Parkinson's disease [13], bipolar disorder [14], autism [15], and schizophrenia [16, 17].

The MI depletion hypothesis of bipolar disorder is still attracting substantial interest because it provides a mechanistic foundation for the action of mood stabilizers [14]; however, attempts to determine MI levels in animal models of, and patients with, bipolar disorder resulted in mixed findings [18-21]. Studies using magnetic resonance spectroscopy (MRS) revealed increased levels of $\mathrm{MI}$ in the frontal cortex and anterior cingulate of juvenile patients with bipolar disorder [21, 22], and normal MI levels in the brain of unmedicated adult bipolar patients [23]. Further, mood stabilizers have been shown to decrease or increase MI in gray matter of the brain of rats and patients with bipolar disorder [24-26].

Conflicting conclusions were also made with respect to the role of MI in schizophrenia. MRS imaging studies showed a reduction of $\mathrm{MI}$ in the anterior cingulate and medial prefrontal cortex [16,27], increased MI in the parietal white matter [28], and no changes in frontal white matter in patients with schizophrenia compared with age-matched controls [29].

Association of Myoinositol Transporters with Schizophrenia and Bipolar Disorder
The reason for the reported inconsistency in MI levels in both bipolar disorder and schizophrenia is unknown. However, the patterns of changes of MI in bipolar disorder and schizophrenia appear to be dependent on the brain region. Therefore, the discrepancies in MI levels may arise from distinct differences in expression and regulation of the MI transporters SMIT/HMIT in different brain areas and specific cell types.

The human SMIT1 gene (SLC5A3) is located at chr21: 3544870-35478561 [30], and was found to be overexpressed in human Down syndrome and in trisomy 16 (Ts16) fetal mice $[31,32]$. The expression of SLC5A3 mRNA in neutrophils was decreased in bipolar disorder type I patients with chronic lithium treatment compared to unmedicated bipolar disorder type I patients and controls [33].

The SMIT2 gene (SLC5A11) is located at chr16: $24,857,184-24,922,949$ and there have been no reports to date on genetic associations of this gene with neuropsychiatric disorders. The HMIT gene (SLC2A13) is located at chr12:40,343,293-40,499,661 and was previously associated with Parkinson's disease [13].

Together, these studies suggest a potential role of altered brain MI levels as well as expression of MI transporters in the pathophysiology of psychiatric disorders. However, there are currently no definitive experiments regarding the precise role and/or potential mechanisms for such a role. Therefore, the aim of this study was to gain a better and clearer view of the precise role of MI transporters in psychiatric disorders. To achieve this goal, we first used available datasets describing the distribution in mouse and human brain of the different MI transporters and the changes in expression of these transporters in the brains of patients with specific psychiatric disorders. We then used an Slc5a3 knockout (KO) mouse model to determine the behavioral phenotype of Slc5a3 germline deletion.

\section{Methods}

Spatial Gene Expression of SLC5A3, SLC5A11, and SLC2A13 in Human Brain

The Genotype-Tissue Expression (GTEx) database was queried for cross-tissue comparisons of the significant findings. The GTEx project is an ongoing effort to build a comprehensive public resource to study tissue-specific gene expression and regulation [34]. Samples were collected from 53 non-diseased tissue sites across nearly 1,000 individuals, primarily for molecular assays including whole genome and exome sequencing, and RNA-Seq. The GTEx portal provides open access to data including gene expression, quantitative trait loci (QTLs), and histology images. The current

Mol Neuropsychiatry 2019;5:200-211 201 
release is V7 including 11,688 samples, 53 tissues, and 714 donors. Because GTEx does not report any data for SLC5A3 human expression, the transcriptional profiles of SLC5A3 in various brain regions were acquired from the anatomically comprehensive atlas of the adult human brain transcriptome (Allen Brain Institute for Brain Science; http://human.brain-map.org) [35, 36].

To further explore the cell specificity and diversity of these genes in the human brain, the single nucleus RNA-Seq data set was queried (http://celltypes.brain-map.org/rnaseq) [37]. This dataset is generated from intact nuclei obtained from frozen human brain tissues to screen cell type diversity in the human middle temporal gyrus (MTG). In total, 15,928 nuclei from 8 human tissue donors ranging in age from 24 to 66 years were analyzed. Analysis of these transcriptional profiles reveals approximately 75 transcriptionally distinct cell types, subdivided into 45 inhibitory neuron types, 24 excitatory neuron types, and 6 non-neuronal types.

Spatial Gene Expressions of Slc5a3, Slc5a11, and Slc2a13 in Mouse Brain

We visually examined raw data from the Allen Brain Atlas, which constitute in situ hybridization section images from different section coordinates in adult mouse brains (Allen Brain Atlas; http://mouse.brain-map.org) [38]. In situ hybridization images from corresponding levels available for the expression of Slc5a3 mRNA (Probe: RP_Baylor_103192), Slc5a11 mRNA (Probe: RP_050126_02_F02), and Slc2a13 (Probe: RP_050524_03_H06 and RP_060315_05_B10) were compared. To further investigate the cell specificity of these genes in the brain, a recent paper using a single-cell nuclear RNA-Seq study was queried [39].

\section{Transporter Gene Expression in Psychiatric Disorders GEO Analyses}

We screened publicly available gene expression studies of genes encoding MI transporters SLC5A3, SLC5A11 and SLC2A13 in the context of schizophrenia and bipolar disorder to obtain human differential expression data. The most comprehensive repository of such data, Gene Expression Omnibus (GEO), returned $7 \mathrm{cu}$ rated studies with differential gene expression (RNA microarray) data from individuals with the abovementioned conditions and healthy controls: GSE4036, GSE12654, GSE21935, GSE12679, GSE5389, GSE5388, and GSE17612. GEO2R with default settings was used to calculate differential expression; of these only GSE21935, GSE12679, GSE5389, and GSE5388 showed significant differential expression (see online suppl. Table S1 for the main features of these datasets).

\section{CommonMind Data Analysis}

The CommonMind Consortium dataset initially describes RNA-Seq data results from schizophrenia $(n=258)$ and controls $(n=279)$ [40]. The dataset also contains bipolar disorder patient samples $(n=47)$ obtained through the CommonMind Consortium Knowledge Portal [40].

\section{Behavioral Studies on Slc5a3 KO Mice}

Animals

The Slc5a3 knockout mouse line was a kind gift from Dr. Gerard Berry (Harvard University) and was generated as previously described $[20,28]$. MI was added to the maternal drinking water (4\%) in the prenatal and postnatal stages of all homozygous mice (to ensure pup survival) and to a subgroup of these mice after weaning (Slc5a3 $\mathrm{KO}+\mathrm{MI})$ to the end of the experiment. All mice used in this study were generated from heterozygous crosses. A subgroup of wild-type (WT) mice was also administered MI in drinking water (4\%) from weaning to the end of the experiment. Ten-week-old male mice were tested with a battery of behavioral paradigms in the following order: locomotion activity, marble burying, T-maze, social interaction, forced swimming, prepulse inhibition (PPI), and contextual fear conditioning. The sequence of specific assays spaced by an interassay interval of 3-6 days was adapted from previously published reports $[41,42]$.

Behavioral Analyses

Locomotor Activity. Locomotor activity was performed as previously described [43]. Mice were placed into a locomotion test chamber (Med Associates, Inc.), and the horizontal activity was recorded for $60 \mathrm{~min}$ and analyzed by Activity Monitor 5 software (Med Associates, Inc.).

Social Interaction. The social interaction assay was performed using the three-chambered box apparatus as we previously described [43]. Two empty wire mesh containment cups were placed in the middle of the right or left chamber (one per each side). The duration of direct contacts between the subject mice with both cups were recorded individually and analyzed by ANY-MAZE software (Stoelting Co.).

PPI Assay. The PPI assay was measured as we previously described [44]. Mice were placed in the startle chambers for 5-min acclimation with $65-\mathrm{dB}$ background noise. The value of PPI was calculated as a percentage score for each of acoustic prepulse intensity: \% PPI $=100-([($ startle response for prepulse + pulse trials)/(startle response for pulse-alone trials) $] \times 100)$. The magnitude of the response was calculated as the average response to all of the startle or prepulse trials.

Forced Swim Test. The forced swim assay was performed as previously described [43]. Mice were placed individually in a transparent glass cylinder containing water at $23-25^{\circ} \mathrm{C}$ and forced to swim. The mice were videotaped for $6 \mathrm{~min}$, and the immobility time was recorded for the last $4 \mathrm{~min}$. ANY-MAZE software was used to record and analyze immobility (Stoelting Co.).

Contextual Fear Conditioning Assay. The contextual fear conditioning assay was performed as previously described [43]. After 3 days of mouse handling, the mice were placed in the conditioning chamber (TSE Systems, Inc.) and received foot shock lasting for 2 s; $24 \mathrm{~h}$ after shock, the mice were placed back into the same chamber (same conditioned context: wallpaper with striped pattern) in the absence of shock for $5 \mathrm{~min}$ and their freezing behavior was assessed during this period (retention session). Freezing behavior was scored as freezing (1) or not (0) within an interval of $5 \mathrm{~s}$ and the percentage of freezing behavior was calculated as $100 \times$ (the number of intervals of freezing/total intervals).

\section{Data Analysis}

Animal Studies. statistical analyses were carried out using GraphPad Prism (GraphPad Software, Inc.). Data are presented as means \pm SEM. Results were analyzed by the Student $t$ test or ANOVA followed by the appropriate post hoc comparisons, and $p<$ 0.05 was considered statistically significant.

Human Study. Differential expression between patients and controls in the CommonMind DLPFC data was computed using the limma R package along with following covariates of RNA integrity, library batch, institution (brain bank), diagnosis, age of 


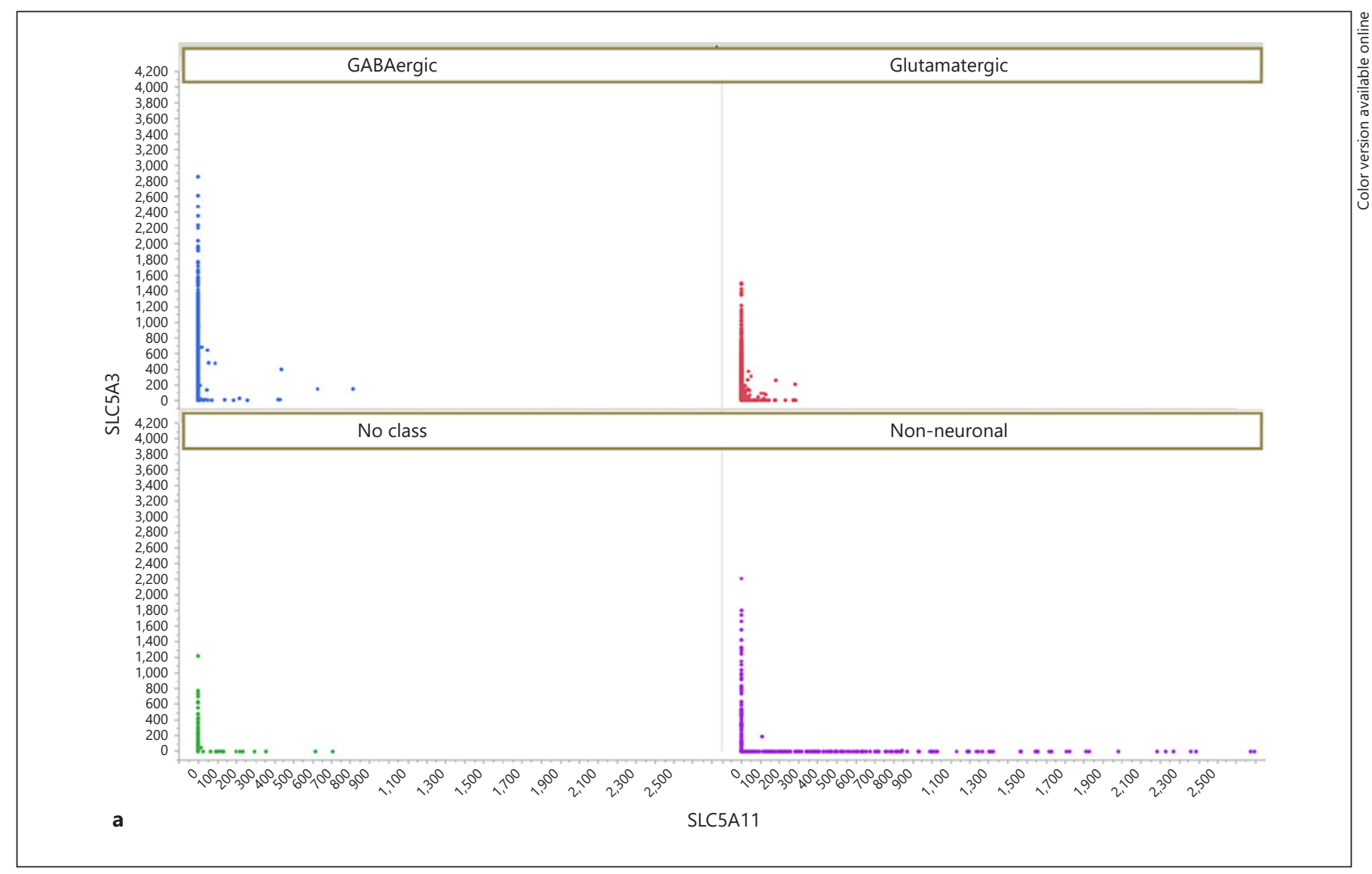

Fig. 1. Expression of MI transporters in single nuclei RNASeq in middle temporal gyrus. a Scatterplot for SLC5A3 and SLC5A11 shows little co-expression as evidenced by the absence of data except along each axis. Expression of SLC5A3 is found predominantly in neuronal nuclei and SLC5A11 is largely non-neuronally expressed as shown by color legend. b SLC5A3 and SLC2A13 show

death, genetic ancestry, postmortem interval, and sex, which explain a substantial fraction (0.42) of the average variance of gene expression being used to adjust the values for all analyses [40]. Differential expression between patients and controls in GEO data was computed using empirical Bayes moderated $t$-statistics test performed by the limma R package.

\section{Results}

\section{Transporter Gene Expression in Human and \\ Mouse Brain}

Gene Expression Distribution in the Human Brain

The GTEx database was queried for cross-tissue comparisons of the significant findings. It shows clearly that SLC5A11 and SLC2A13 are highly expressed in multiple co-expression as evidenced by the presence of data points showing overlap in each nucleus for neuronal nuclei, and a minority of cells tested positive for both RNA expression. The regression line fit is shown by $R^{2}$ values that were highly significant for co-expression in neurons. c Scatterplot for each lamina in MTG. SLC5A3 and SLC2A13 show co-expression in all laminae.

(Figure continued on next page.)

brain regions compared to other tissues (online suppl. Fig. S1.a, b; for all online suppl. material, see www.karger. com/doi/10.1159/000501125) [34]. Because GTEx does not report any data for SLC5A3 human expression, the Allen Brain Institute for Brain Science database was queried for brain regional expression of SLC5A3, and the results show that $S L C 5 A 3$ is differentially expressed in various brain regions, with the highest expression being in the choroid plexus (online suppl. Fig S1.c) [36].

The cellular specificity of SLC5A3, SLC5A11, and SLC2A13 were compared across broad classes (GABA, glutamate, non-neuronal, and no class) as well as cell clusters. In 15,928 human nuclei sampled in the MTG, there was scant evidence for the co-expression of SLC5A3 and SLC5A11 (Fig. 1a; online suppl. Fig. S2.a) or the co-expression 


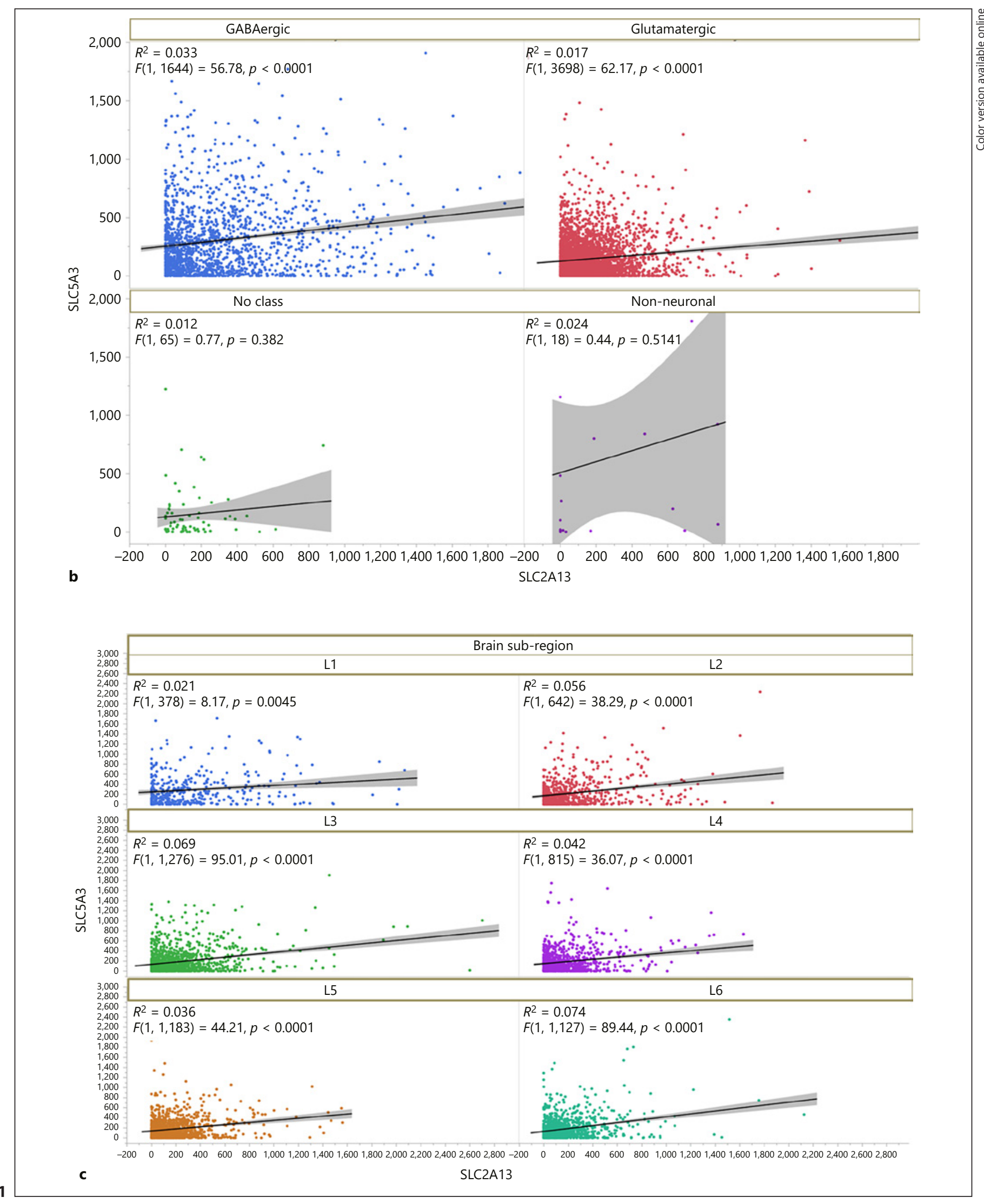


Table 1. Gene expression of SLC5A3, SLC5A11, and SLC2A13 in schizophrenia and bipolar disorder (CommonMind)

a CommonMind schizophrenia compared to controls

\begin{tabular}{llllll}
\hline Gene/isoform & Symbol & Ensembl ID & Average expression & $\log _{2}$ fold change & $p$ value \\
\hline SLC5A3 & SLC5A3 & ENSG00000198743 & 7.622 & 0.168 & 0.001034 \\
SLC5A11 & SLC5A11 & ENSG00000158865 & 2.949 & -0.0353 & 0.03806 \\
SLC2A13 & SLC2A13 & ENSG00000151229 & 8.378 & 0.1029 & 0.6583 \\
\hline
\end{tabular}

b CommonMind bipolar disorder compared to controls

\begin{tabular}{llllll}
\hline Gene/isoform & Symbol & Ensembl ID & Average expression & $\log _{2}$ fold change & $p$ value \\
\hline SLC5A3 & SLC5A3 & ENSG00000198743 & 7.580 & 0.05444 & 0.56354 \\
SLC5A11 & SLC5A11 & ENSG00000158865 & 3.269713 & 0.47287 & 0.833295 \\
SLC2A13 & SLC2A13 & ENSG00000151229 & 8.394 & 0.16632 & 0.001622 \\
\end{tabular}

RNA-Seq data results from schizophrenia $(n=258)$, bipolar disorder $(n=47)$, and controls $(n=279)$.

of SLC5A11 and SLC2A13 (online suppl. Fig. S2.b). SLC5A11 showed clear non-neuronal expression and was highest in oligodendrocytes in layers 1-6 of oligodendrocyte precursor cells' positive nuclei (online suppl. Fig. S2.a, b). Single nuclei in the MTG generally co-expressed SLC5A3 and SLC2A13: of 15,928 snRNA-Seq nuclei there were $5,421 \mathrm{SLC} \mathrm{A} 3^{+} / \mathrm{SLC} 2 \mathrm{~A} 13^{+}$nuclei distributed across 6 cortical layers (Fig. 1b, c; online suppl. Fig. S2.c). The regression lines for co-expression were highly significant (shown in Fig. $1 \mathrm{~b} ; p$ value range from layer $1 p=4 \mathrm{E}-03$ to layer $3 p=1 \mathrm{E}-21)$. Most cells that co-expressed both SLC$5 \mathrm{~A} 3$ and SLC2A13 were either GABAergic ( $p$ value for regression $4.1 \mathrm{E}-15$ ) or glutamatergic ( $p$ value for regression 6.8E-15, Fig. 1b; online suppl. Fig. S2.c). A total of 83 non-neuronal nuclei co-express SLC5A3 and SLC2A13 (Fig. 1b; online suppl. Fig. S2.c), while GABAergic $(1,644)$ and glutamatergic $(3,698)$ nuclei co-express both genes.

Gene Expression Distribution in the Mouse Brain

Similar to what we found in the human brain, the gene expression map of the three MI transporters in the mouse brain shows distinct topographical distribution (online suppl. Fig. S3). In situ hybridization data downloaded from the Allen Brain Atlas show the distinct distribution of the three transporters (online suppl. Fig. S3). For this analysis, the expression value reported at the website was used. SLC5A11 transcript expression was relatively low in all regions of the mouse brain, although we were previously able to detect SLC5A11 protein expression, highly localized to the axon initial segment, in mouse hippocampal neurons [45]. It is also evident that the levels of SLC$2 \mathrm{~A} 13$ transcript expression are higher than those of SLC$5 \mathrm{~A} 3$ in most brain regions, and that the hippocampus, cerebral cortex, and hypothalamus contain the highest levels of SLC5A3.

\section{Analysis of Gene Expression in Schizophrenia and}

Bipolar Disorder CommonMind Consortium and GEO Studies

We used a gene expression database that provides RNA sequencing data in the postmortem human dorsolateral prefrontal cortex of individuals with schizophrenia, bipolar disorder, and control subjects (CommonMind Consortium) and examined the data for SLC5A3, SLC5A11, and SLC2A13 expression. SLC5A3 was significantly increased between schizophrenia and control individuals (SLC5A3, ENSG00000198743, $p=0.038$ FDR corrected, $\log _{2}$ difference $\left.=0.16\right)$ and showed a trend for SLC2A13 (HMIT, ENSG00000151229, $p=0.08$ FDR corrected, $\log _{2}$ difference $=0.10)($ Table 1a). SLC5A11 expression was significantly increased between bipolar disorder and control subjects (SLC5A11, ENSG00000158865, $p=0.0435$ FDR corrected, $\log _{2}$ difference $\left.=0.47\right)($ Table $1 b)$.

In smaller GEO sample sets (online suppl. Tables S1S4), the results were underpowered to replicate the CommonMind Consortium results for schizophrenia and bipolar disorder. The details of those results from multiple GEO datasets were obtained from the dorsolateral pre- 


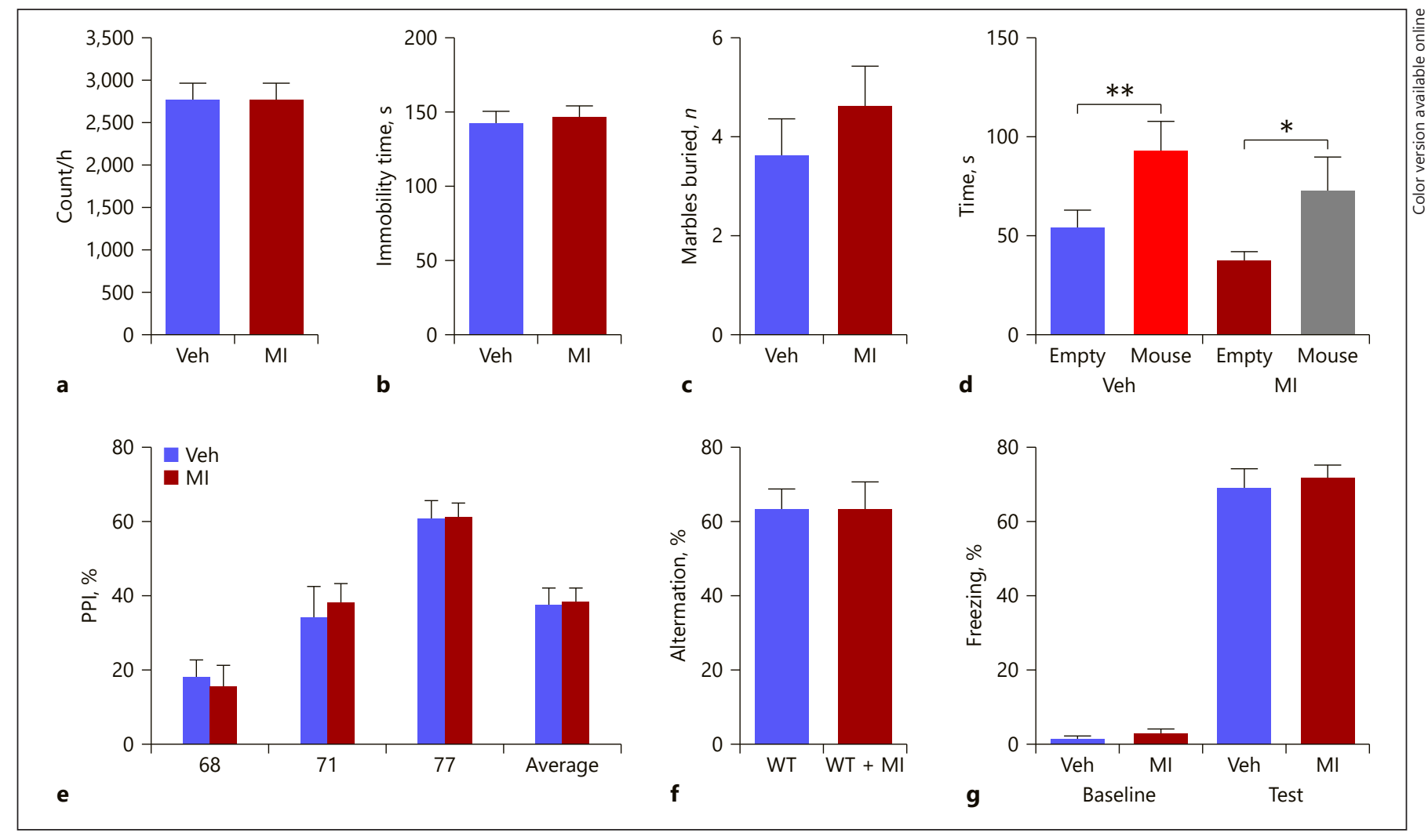

Fig. 2. Behavioral effects of MI in WT mice. a Distance that mice travelled in $60 \mathrm{~min}$ of the locomotion assay; unpaired Student test $(t=0.39, p>0.05, n=10)$ : WT vs. KO. b Immobile time in the forced swimming assay; unpaired Student test $(t=0.39, p=0.6999$, $n=10$ ) revealed no significant drug effect. c Number of marbles buried in $30 \mathrm{~min}$; unpaired Student test $(t=0.909, p=0.375, n=$ 10) revealed no significant drug effect. $\mathbf{d}$ Time mice spent interacting with empty cup and unfamiliar mouse in the social interaction assay; two-way ANOVA revealed a significant object effect $\left(F_{1,30}=\right.$ 21.01, $p=0.004, n=7-10)$ and but no drug effect $\left(F_{1,30}=1.3, p>\right.$

frontal cortex, anterior prefrontal cortex, superior temporal cortex, orbitofrontal cortex, and cerebellum. In GEO dataset (GSE21935), SLC5A3 (SMIT1) expression was significantly increased in the superior temporal cortex (BA22) in schizophrenia patients compared to controls $($ FDR $p$ value $=0.042)$. The aforementioned data were obtained using two SLC5A3 probe sets: 212944_at and 213164_at; these probe sets are given annotation grade A.

\section{Animal Studies}

SLC5A3 KO mice displayed locomotor activity comparable to that of the WT group. While chronic administration of MI did not produce any change in the locomotor activity in the WT mice ( $p>0.05$; Fig. 2a), it signifi-
$0.05)$ followed by Bonferroni post hoc test. ${ }^{*} p<0.05,{ }^{* *} p<0.01$, empty cup vs. unfamiliar mouse. e Prepulse inhibition ratios against three prepulse stimulations in the PPI assay; two-way ANOVA revealed no significant drug effect $\left(F_{1,72}=0.02, p=0.887\right.$, $n=10)$. $\mathbf{f}$ Percentage of the alternation choice mice made in the $\mathrm{T}$ maze spontaneous assay; unpaired Student test $(t=0.39, p>0.05$, $n=10)$. g Percentage of freezing behavior in contextual fear conditioning assay; two-way ANOVA revealed no significant drug effect $\left(F_{1,36}=0.475, p=0.344, n=10\right)$ followed by Bonferroni post hoc test. Data are presented as means \pm SEM. Veh, vehicle. cantly increased the locomotor activity in the SLC5A3 KO mice ( $p<0.05$; Fig. 3a).

MI did not cause any change in the forced swim test in WT animals ( $p>0.05$; Fig. 2b). SLC5A3 KO mice displayed lower immobility time than WT mice ( $p<0.05$; Fig. $3 b)$, suggesting that SLC5A3 $\mathrm{KO}$ mice have higher manic behavior, but MI was unable to reverse the manic-like behavior observed in SLC5A3 KO mice ( $p>0.05$; Fig $3 b$ ).

Repetitive behavior was measured using the marble burying assay. In this test, chronic MI administration did not affect repetitive behavior in the WT animals $(p>0.05$; Fig. 2c); however, it reversed the augmented repetitive behavior in the SLC5A3 KO mice ( $p<0.05$; Fig. 3c). Indeed, MI decreased the number of buried marbles in SLC5A3 

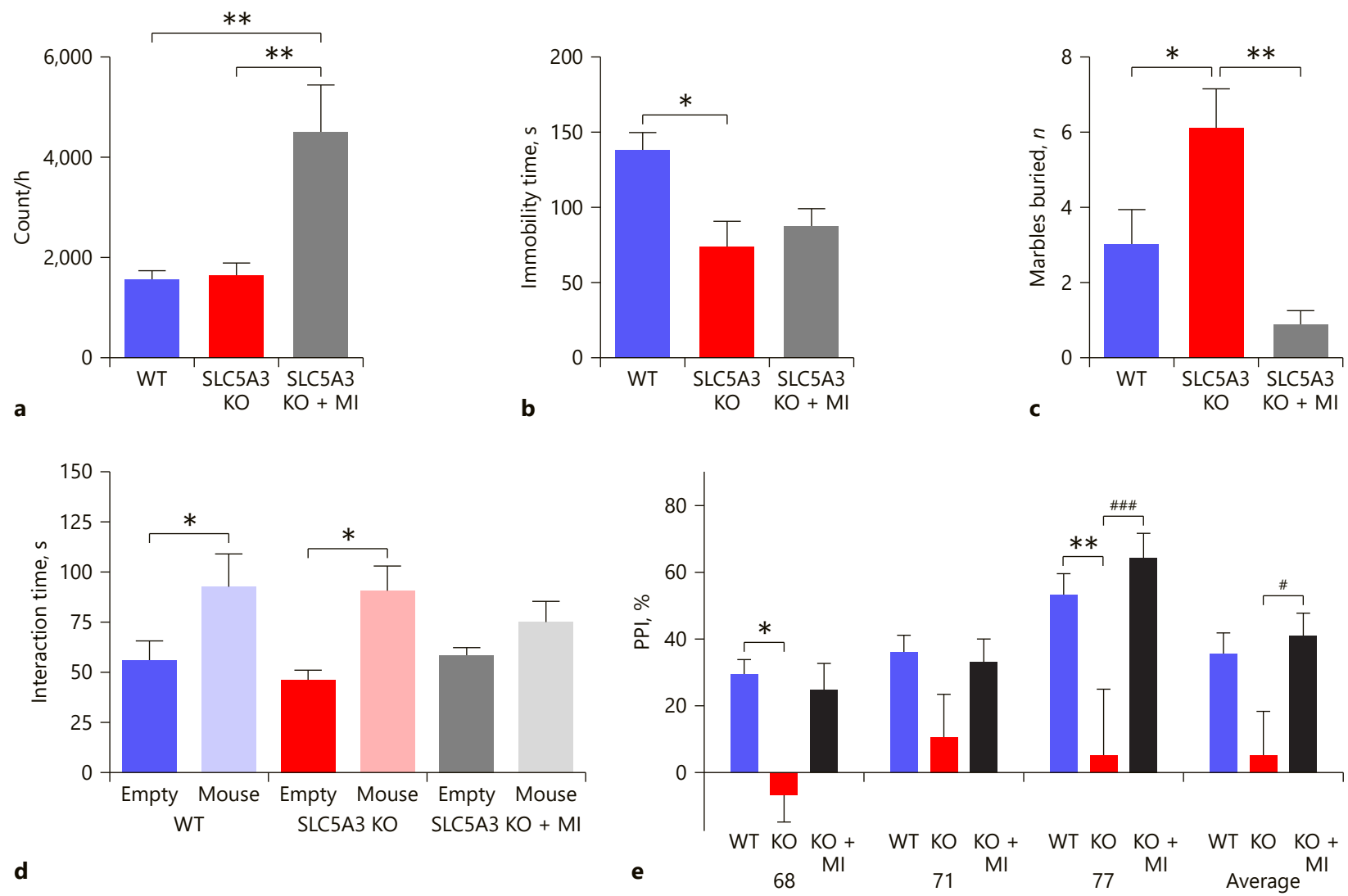

d

e
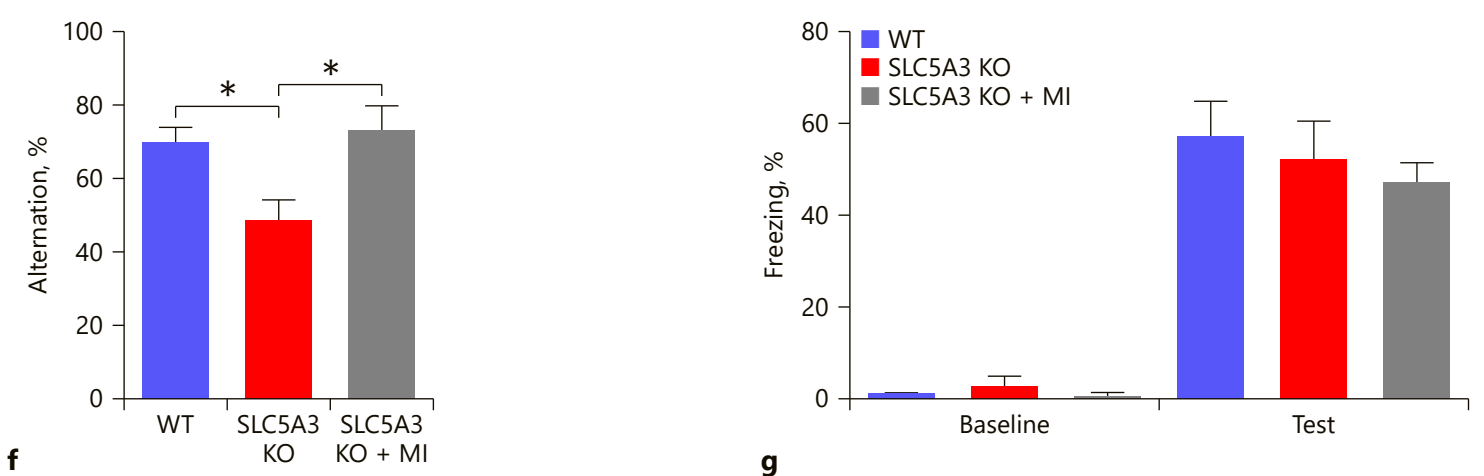

g

Fig. 3. Behavioral phenotype of Slc5a3 $\mathrm{KO}$ mice and following chronic MI treatment. a Distance mice travelled in $60 \mathrm{~min}$ of the locomotion assay; one-way ANOVA followed by Tukey post-test $\left(F_{2,23}=8.36,{ }^{* *} p<0.01, n=8\right)$. b Immobile time in the forced swimming assay; one-way ANOVA revealed a significant genotype effect $\left(F_{2,22}=4.675, p=0.02, n=7-9\right)$ followed by Bonferroni post-test. * $p<0.05$, WT vs. Slc5a3 KO. c Number of marbles buried in $30 \mathrm{~min}$; one-way ANOVA revealed significant genotype and treatment effects $\left(F_{2,23}=9.950, p=0.0009, n=8\right)$ followed by Bonferroni post-test. ${ }^{*} p<0.05,{ }^{* * *} p<0.001$. d Time mice spent interacting with empty cup and unfamiliar mouse in the social interaction assay; two-way ANOVA revealed a significant object effect $\left(F_{1,46}=14.32, p=0.0004, n=8-9\right)$ but not object $\times$ treatment interaction $\left(F_{2,46}=0.8323, p>0.05\right)$ followed by Bonferroni post hoc test. ${ }^{*} p<0.05$, empty cup vs. unfamiliar mouse. Data are presented as means \pm SEM. e Prepulse inhibition ratios against three prepulse stimulations in the PPI assay; one-way ANOVAs revealed a significant genotype and treatment effect $(F=4.8, p<0.0001$, $n=7-9$ ) followed by Bonferroni post-test. ${ }^{*} p<0.05$, ${ }^{* *} p<0.01$, WT vs. KO; ${ }^{\#} p<0.05,{ }^{\# \#} p<0.001, \mathrm{KO}$ vs. KO + MI. f Percentage of the alternation choice mice made in the T-maze spontaneous assay; one-way ANOVA revealed a significant effect of the genotype and treatment $\left(F_{2,20}=5.472, p=0.013, n=7\right) .{ }^{*} p<0.05$. Data are presented as means \pm SEM. $g$ Percentage of freezing behavior in contextual fear conditioning assay; two-way ANOVA revealed no significant genotype or treatment effect $\left(F_{2,42}=0.468, p=0.344\right.$, $n=7-9)$ followed by Bonferroni post hoc test. Data are presented as means \pm SEM. 
KO mice to levels that were even lower than in WT mice (albeit not significant compared with WT, $p=0.057$ ).

In the social interaction assay, both WT and SLC5A3 KO mice displayed more interaction with the unfamiliar mouse than with the empty cup ( $p<0.05$; Fig. $3 \mathrm{~d}$ ). The WT-MI group spent more time interacting with the unfamiliar mouse than with the empty cup ( $p<0.05$; Fig. 2 d), however, the SLC5A3 KO-MI group displayed a deficit in sociability, and spent a similar amount of time with the unfamiliar mouse and the empty cup ( $p>0.05$; Fig. 3d).

We then studied the effect of deletion of SLC5A3 and the MI treatment on the sensorimotor gating response. SLC5A3 KO mice exhibited a significant decrease in PPI ratios ( $p<0.05$; Fig. $3 \mathrm{e}$ ), suggesting an impairment in sensorimotor gating function. While chronic MI treatment did not produce any effect on the PPI ratios in the WT animals ( $p>0.05$; Fig 2e), it reversed the PPI deficit in the SLC5A3 KO group, restoring PPI ratios to normal values $(p<0.05$; Fig. 3e).

In the spontaneous T-maze alternation assay which measures working memory, MI did not have any effect on the percentage of arm choice alternation in the WT mice ( $p>0.05$; Fig. 2f). SLC5A3 KO mice showed a decrease in the percentage of arm choice alternation compared to the WT mice ( $p<0.05$; Fig. 3f). Chronic MI administration reversed the memory deficit and increased the percentage of arm choice alternation in the SLC5A3 KO mice to the levels of WT mice ( $p>0.05$; Fig. 3f).

In the contextual fear conditioning assay, all groups barely exhibited freezing behavior, with no significant effect of the genotype or the MI treatment before the foot shock stimulus during the training session, and all groups exhibited a similar level of increase in the freezing behavior percentage in the retention session $(p>0.05$; Fig. $2 \mathrm{~g}$, $3 g)$. Together, these results suggest that SLC5A3 KO mice display cognitive deficits in working memory but not long-term contextual memory, and that MI improves the impaired but not the normal working memory.

\section{Discussion}

Several lines of evidence from animal and human studies have linked MI with the pathophysiology and/or treatment of psychiatric disorders such as schizophrenia, autism, depression, ADHD, bipolar disorder, and OCD. Recent work showed the phosphatidylinositol signaling system is a highly ranked pathway in cross-disorder comparisons of schizophrenia, autism spectrum disorder, and bipolar disorder using a Deep Structured Phenotype Net- work analysis and other machine learning algorithms [46]. Thus, we studied MI transport in schizophrenia and bipolar disorder choosing three transporters of MI that differentially regulate the level of MI (SMIT1, SMIT2 and HMIT, encoded by SLC5A3, SLC5A11, and SLC2A13, respectively) due to varying transport kinetics (capacity and affinity). Our current study reveals that the distinct roles of MI in schizophrenia and bipolar disorder may reflect the regional disparity, cell-specific expression patterns, and/or dynamic control of MI transporters. In this context, we further studied specific genetic KO of one transporter (SMIT1; Slc5a3) to help determine the eventual behavioral phenotypes and/or symptomatic pattern in an animal model of psychiatric disorders.

Our analysis of existing datasets of gene expression in normal brain demonstrates that SLC5A3, SLC5A11, and SLC2A13 exhibit distinct regional expression in the human brain. Of interest, individual neurons appear to utilize SLC5A3 and SLC2A13 together while SLC5A11 appears to be largely non-neuronal. These cell-specific patterns can thus partially account for different uptake levels of MI and concordance to downstream second messenger signaling pathways. Analysis of the large CommonMind RNA-Seq dataset revealed increased SLC5A3 in schizophrenia and increased SLC5A11 in the dorsolateral prefrontal cortex in bipolar disorder. GEO results showed both increased and decreased SLC5A3 expression in schizophrenia depending on the brain regions and microarray probe sets. While these results may help explain the inconsistency of previous data on the role of MI in psychiatric disorders, they also suggest that altered expression or function of one or more of these transporters might be associated with specific regional expression differences and have specificity to a handful of phenotypes.

Using Slc5a3 KO mice, those phenotypes were tested in a series of behavioral assays that correspond to symptoms of psychiatric disorders known to be associated with abnormal MI levels including schizophrenia, depression, and bipolar disorder. While Slc5a3 KO mice exhibited normal locomotor activity, social behavior, and contextual memory, these animals displayed excessive repetitive behavior, increased mania-like behavior, impaired working memory, and deficits in prepulse inhibition. SMIT1 (SLC5A3) is only one of three active transporters that regulate $\mathrm{MI}$ in the brain. Given the distinctive brain distribution, cellular expression, and various capacities and affinities of the three different MI transporters, it is conceivable that the ultimate behavioral phenotype induced by SLC5A3 deletion is determined by the specificity and topographical extent of the contributory mechanisms of 
the other two transporters, encoded by SLC5A11 and SLC$2 \mathrm{~A} 13$, in response to SLC5A3 deletion. First, SLC5A11 is largely non-neuronal while SLC5A3 is neuronal, so SLC$5 \mathrm{~A} 11$ is unlikely to rescue functions ascribed to neuronal control in the Slc5a3 KO. It is possible that SLC2A13 could be compensatory in the same cells where SLC5A3 is lost. This argument is supported by our observation of highly significant patterns of co-expression in multiple layers of cortex for SLC5A3 and SLC2A13. However, the discrepancy in the brain regional distribution of SLC5A3 and SLC2A13 suggests that SLC2A13 can only compensate for SLC5A3 absence in specific brain regions (where SLC2A13 is highly expressed). Therefore, the pattern and magnitude of specific behavioral deficits induced by SLC$5 \mathrm{~A} 3$ depletion may be mitigated by the ability of SLC$2 \mathrm{~A} 13$ to compensate for the loss of SLC5A3 in brain regions that express SLC2A13.

While chronic MI administration in drinking water did not induce effects on any of the behavioral responses in WT mice, its effects on Slc5a3 KO mice can be divided into three categories: (1) no effect on the behavioral responses of Slc5a3 KO mice such as those quantified by immobility in the forced swim test; (2) reversal of behavioral deficits in the Slc5a3 KO mice such as those in repetitive behavior, working memory impairment, and the prepulse inhibition deficit; and (3) induction of distinct behaviors, such as increase in locomotor activity and decrease in social interaction which mirror positive and negative symptoms of schizophrenia, respectively. Chronic dietary inositol is known to differentially elevate inositol levels in different regions of the brain, e.g., 36 and $27 \%$ increases in the cortex and hippocampus, respectively, with no effect on inositol levels in the striatum or cerebellum [47]. In the light of these data, our results showing the lack of significant impacts of mild dietary MI supplementation on behavioral phenotypes of WT mice are not surprising.

Slc5a3 KO mice exhibited deficits in prepulse inhibition and spatial working memory, as well as increased repetitive behavior, all of which involve activation of the cortico-striatal-thalamo-cortical (CSTC) circuit [48, 49]. Chronic MI administration reversed these behavioral deficits, suggesting that MI was taken up through other transporters (most likely Slc2a13) into the neurons or astrocytes in the CSTC circuit. Imbalances of the CSTC circuit are associated with a number of neuropsychiatric and neurological disorders, including OCD, bipolar disorder, schizophrenia, and ADHD [49-51]. Interestingly, our analysis of the anatomical distribution of MI transporter genes reveals that SLC5A3, which is highly expressed in the mouse striatum (the input nucleus to the basal ganglia), is scarcely expressed in the human striatum. However, in contrast to mouse thalamus, human thalamus contains high levels of SLC5A3. In addition, both the mouse and the human cortex contain high levels of SLC$5 \mathrm{~A} 3$. Together, these data suggest that although speciesspecific regional expression differences likely occur, SLC$5 \mathrm{~A} 3$ plays a significant role in the regulation of some regions of the CSTC circuit in both mice and humans and, therefore, might be involved in the pathophysiology of psychiatric disorders that are associated with the dysfunctions of the CSTC circuit.

Our data support a correlation between the differential expression levels of MI transporters and schizophrenia and bipolar disorder, suggesting that brain region-specific aberration of one or more of these transporters can induce behavioral phenotypes and/or symptomatic pattern of these disorders. Our data also suggest MI transporters as potential therapeutic targets for the treatment of schizophrenia and bipolar disorder. Further studies are necessary to determine the behavioral phenotypes associated with $\operatorname{Slc2a} 13$ genetic deletion in mice and to a lesser extent of Slc5a11. The remaining question is how an increased expression of SLC5A3 and SLC2A13 in a regional-specific manner that occurs in human neuropsychiatric disorders may also determine potential regulators of MI transporters and whether those are differentially regulated within different neurotransmitter systems by epigenetic and environmental factors.

\section{Acknowledgments}

This work was supported by the Eric L. and Lila D. Nelson Chair in Neuropharmacology (to O.C.), the US National Institutes of Health, National Institute of General Medical Sciences (GM115189 and GM130377 to G.W.A.) and National Institute of Mental Health (MH085801 to M.P.V.) and Pritzker Neuropsychiatric Disorders Research Consortium (to M.P.V.).

\section{Statement of Ethics}

All experimental procedures were approved by the Institutional Animal Care and Use Committee of University of California, Irvine and were performed in compliance with national and institutional guidelines for the care and use of laboratory animals.

\section{Disclosure Statement}

The authors declare no conflict of interest. 


\section{References}

1 Strange K, Emma F, Paredes A, Morrison R. Osmoregulatory changes in myo-inositol content and $\mathrm{Na}+$ /myo-inositol cotransport in rat cortical astrocytes. Glia. 1994 Sep;12(1): 35-43.

2 Silver SM, Schroeder BM, Sterns RH, Rojiani AM. Myoinositol administration improves survival and reduces myelinolysis after rapid correction of chronic hyponatremia in rats. J Neuropathol Exp Neurol. 2006 Jan;65(1):3744.

3 Bissonnette P, Lahjouji K, Coady MJ, Lapointe JY. Effects of hyperosmolarity on the $\mathrm{Na}+$ -myo-inositol cotransporter SMIT2 stably transfected in the Madin-Darby canine kidney cell line. Am J Physiol Cell Physiol. 2008 Sep;295(3):C791-9.

4 Shirayama Y, Takahashi M, Osone F, Hara A, Okubo T. Myo-inositol, Glutamate, and Glutamine in the Prefrontal Cortex, Hippocampus, and Amygdala in Major Depression. Biol Psychiatry Cogn Neurosci Neuroimaging. 2017 Mar;2(2):196-204.

5 Einat H, Belmaker RH, Zangen A, Overstreet $\mathrm{DH}$, Yadid G. Chronic inositol treatment reduces depression-like immobility of Flinders Sensitive Line rats in the forced swim test. Depress Anxiety. 2002;15(3):148-51.

6 Carey PD, Warwick J, Harvey BH, Stein DJ, Seedat S. Single photon emission computed tomography (SPECT) in obsessive-compulsive disorder before and after treatment with inositol. Metab Brain Dis. 2004 Jun;19(1-2): 125-34.

7 Fux M, Levine J, Aviv A, Belmaker RH. Inositol treatment of obsessive-compulsive disorder. Am J Psychiatry. 1996 Sep;153(9):121921.

8 Palatnik A, Frolov K, Fux M, Benjamin J. Double-blind, controlled, crossover trial of inositol versus fluvoxamine for the treatment of panic disorder. J Clin Psychopharmacol. 2001 Jun;21(3):335-9.

9 Benjamin J, Levine J, Fux M, Aviv A, Levy D, Belmaker RH. Double-blind, placebo-controlled, crossover trial of inositol treatment for panic disorder. Am J Psychiatry. 1995 Jul; 152(7):1084-6.

10 Tafazoli S, O’Neill J, Bejjani A, Ly R, Salamon $\mathrm{N}, \mathrm{McCracken} \mathrm{JT}$, et al. $1 \mathrm{H}$ MRSI of middle frontal gyrus in pediatric ADHD. J Psychiatr Res. 2013 Apr;47(4):505-12.

11 Voevodskaya O, Sundgren PC, Strandberg O, Zetterberg H, Minthon L, Blennow K, et al.; Swedish BioFINDER study group. Myo-inositol changes precede amyloid pathology and relate to APOE genotype in Alzheimer disease. Neurology. 2016 May;86(19):1754-61.

12 Zhu X, Schuff N, Kornak J, Soher B, Yaffe K, Kramer JH, et al. Effects of Alzheimer disease on fronto-parietal brain $\mathrm{N}$-acetyl aspartate and myo-inositol using magnetic resonance spectroscopic imaging. Alzheimer Dis Assoc Disord. 2006 Apr-Jun;20(2):77-85.
13 Foo JN, Tan LC, Irwan ID, Au WL, Low HQ, Prakash KM, et al. Genome-wide association study of Parkinson's disease in East Asians. Hum Mol Genet. 2017 Jan;26(1):226-32.

14 Shimon H, Agam G, Belmaker RH, Hyde TM, Kleinman JE. Reduced frontal cortex inositol levels in postmortem brain of suicide victims and patients with bipolar disorder. Am J Psychiatry. 1997 Aug;154(8):1148-50.

15 Levine J, Aviram A, Holan A, Ring A, Barak Y, Belmaker RH. Inositol treatment of autism. J Neural Transm (Vienna). 1997;104(2-3): 307-10.

16 Das TK, Dey A, Sabesan P, Javadzadeh A, Théberge J, Radua J, et al. Putative Astroglial Dysfunction in Schizophrenia: A Meta-Analysis of 1H-MRS Studies of Medial Prefrontal Myo-Inositol. Front Psychiatry. 2018 Sep;9: 438.

17 Chiappelli J, Rowland LM, Wijtenburg SA, Muellerklein F, Tagamets M, McMahon RP, et al. Evaluation of Myo-Inositol as a Potential Biomarker for Depression in Schizophrenia. Neuropsychopharmacology. 2015 Aug;40(9): 2157-64.

18 Jadhav S, Russo S, Cowart LA, Greenberg ML. Inositol Depletion Induced by Acute Treatment of the Bipolar Disorder Drug Valproate Increases Levels of Phytosphingosine. J Biol Chem. 2017 Mar;292(12):4953-9.

19 Forester BP, Finn CT, Berlow YA, Wardrop M, Renshaw PF, Moore CM. Brain lithium, $\mathrm{N}$-acetyl aspartate and myo-inositol levels in older adults with bipolar disorder treated with lithium: a lithium-7 and proton magnetic resonance spectroscopy study. Bipolar Disord. 2008 Sep;10(6):691-700.

20 Silverstone PH, McGrath BM, Kim H. Bipolar disorder and myo-inositol: a review of the magnetic resonance spectroscopy findings. Bipolar Disord. 2005 Feb;7(1):1-10.

21 Cecil KM, DelBello MP, Sellars MC, Strakowski SM. Proton magnetic resonance spectroscopy of the frontal lobe and cerebellar vermis in children with a mood disorder and a familial risk for bipolar disorders. J Child Adolesc Psychopharmacol. 2003;13(4):545-55.

22 Davanzo P, Yue K, Thomas MA, Belin T, Mintz J, Venkatraman TN, et al. Proton magnetic resonance spectroscopy of bipolar disorder versus intermittent explosive disorder in children and adolescents. Am J Psychiatry. 2003 Aug;160(8):1442-52.

23 Dager SR, Friedman SD, Parow A, Demopulos C, Stoll AL, Lyoo IK, et al. Brain metabolic alterations in medication-free patients with bipolar disorder. Arch Gen Psychiatry. 2004 May;61(5):450-8.

24 Silverstone PH, Wu RH, O’Donnell T, Ulrich M, Asghar SJ, Hanstock CC. Chronic treatment with both lithium and sodium valproate may normalize phosphoinositol cycle activity in bipolar patients. Hum Psychopharmacol. 2002 Oct; 17(7):321-7.
25 Moore GJ, Bebchuk JM, Parrish JK, Faulk MW, Arfken CL, Strahl-Bevacqua J, et al. Temporal dissociation between lithium-induced changes in frontal lobe myo-inositol and clinical response in manic-depressive illness. Am J Psychiatry. 1999 Dec;156(12): 1902-8.

26 Friedman SD, Dager SR, Parow A, Hirashima F, Demopulos C, Stoll AL, et al. Lithium and valproic acid treatment effects on brain chemistry in bipolar disorder. Biol Psychiatry. 2004 Sep;56(5):340-8.

27 Chiu PW, Lui SS, Hung KS, Chan RC, Chan $\mathrm{Q}$, Sham PC, et al. In vivo gamma-aminobutyric acid and glutamate levels in people with first-episode schizophrenia: A proton magnetic resonance spectroscopy study. Schizophr Res. 2018 Mar;193:295-303.

28 Auer DP, Wilke M, Grabner A, Heidenreich JO, Bronisch T, Wetter TC. Reduced NAA in the thalamus and altered membrane and glial metabolism in schizophrenic patients detected by $1 \mathrm{H}-\mathrm{MRS}$ and tissue segmentation. Schizophr Res. 2001 Oct;52(1-2):87-99.

29 Bustillo JR, Jones T, Chen H, Lemke N, Abbott C, Qualls C, et al. Glutamatergic and Neuronal Dysfunction in Gray and White Matter: A Spectroscopic Imaging Study in a Large Schizophrenia Sample. Schizophr Bull. 2017 May;43(3):611-9.

30 Berry GT, Mallee JJ, Kwon HM, Rim JS, Mulla WR, Muenke M, et al. The human osmoregulatory $\mathrm{Na}+$ /myo-inositol cotransporter gene (SLC5A3): molecular cloning and localization to chromosome 21. Genomics. 1995 Jan;25(2):507-13.

31 Berry GT, Wang ZJ, Dreha SF, Finucane BM, Zimmerman RA. In vivo brain myo-inositol levels in children with Down syndrome. J Pediatr. 1999 Jul;135(1):94-7.

32 Beacher F, Simmons A, Daly E, Prasher V, Adams C, Margallo-Lana ML, et al. Hippocampal myo-inositol and cognitive ability in adults with Down syndrome: an in vivo proton magnetic resonance spectroscopy study. Arch Gen Psychiatry. 2005 Dec;62(12):13605 .

33 Willmroth F, Drieling T, Lamla U, Marcushen M, Wark HJ, van Calker D. Sodium-myoinositol co-transporter (SMIT-1) mRNA is increased in neutrophils of patients with bipolar 1 disorder and down-regulated under treatment with mood stabilizers. Int J Neuropsychopharmacol. 2007 Feb;10(1):63-71.

34 GTEx Consortium. The Genotype-Tissue Expression (GTEx) project. Nat Genet. 2013 Jun;45(6):580-5.

35 Hawrylycz M, Miller JA, Menon V, Feng D, Dolbeare T, Guillozet-Bongaarts AL, et al. Canonical genetic signatures of the adult human brain. Nat Neurosci. 2015 Dec;18(12):183244.

Vawter/Hamzeh/Muradyan/Civelli/ Abbott/Alachkar

DOI: $10.1159 / 00050112$ 
36 Hawrylycz MJ, Lein ES, Guillozet-Bongaarts AL, Shen EH, Ng L, Miller JA, et al. An anatomically comprehensive atlas of the adult human brain transcriptome. Nature. 2012 Sep;489(7416):391-9.

37 Regev A, Teichmann SA, Lander ES, Amit I, Benoist C, Birney E, et al.; Human Cell Atlas Meeting Participants. The Human Cell Atlas. Elife. 2017 Dec;6:e27041.

38 Lein ES, Hawrylycz MJ, Ao N, Ayres M, Bensinger A, Bernard A, et al. Genome-wide atlas of gene expression in the adult mouse brain. Nature. 2007 Jan;445(7124):168-76.

39 Rosenberg AB, Roco CM, Muscat RA, Kuchina A, Sample P, Yao Z, et al. Single-cell profiling of the developing mouse brain and spinal cord with split-pool barcoding. Science. 2018 Apr;360(6385):176-82.

40 Fromer M, Roussos P, Sieberts SK, Johnson JS, Kavanagh DH, Perumal TM, et al. Gene expression elucidates functional impact of polygenic risk for schizophrenia. Nat Neurosci. 2016 Nov; 19(11):1442-53.
41 Paylor R, Spencer CM, Yuva-Paylor LA, Pieke-Dahl S. The use of behavioral test batteries, II: effect of test interval. Physiol Behav. 2006 Jan;87(1):95-102.

42 Mcllwain KL, Merriweather MY, Yuva-Paylor LA, Paylor R. The use of behavioral test batteries: effects of training history. Physiol Behav. 2001 Aug;73(5):705-17.

43 Alachkar A, Wang L, Yoshimura R, Hamzeh AR, Wang Z, Sanathara N, et al. Prenatal onecarbon metabolism dysregulation programs schizophrenia-like deficits. Mol Psychiatry. 2018 Feb;23(2):282-94.

44 Wang L, Alachkar A, Sanathara N, Belluzzi JD, Wang Z, Civelli O. A Methionine-Induced Animal Model of Schizophrenia: Face and Predictive Validity. Int J Neuropsychopharmacol. 2015 May;18(12):pyv054.

45 Neverisky DL, Abbott GW. KCNQ-SMIT complex formation facilitates ion channelsolute transporter cross talk. FASEB J. 2017 Jul;31(7):2828-38.

46 Wang D, Liu S, Warrell J, Won H, Shi X, Navarro FC, et al.; PsychENCODE Consortium. Comprehensive functional genomic resource and integrative model for the human brain. Science. 2018 Dec;362(6420):eaat8464.
47 Kofman O, Agam G, Shapiro J, Spencer A. Chronic dietary inositol enhances locomotor activity and brain inositol levels in rats. Psychopharmacology (Berl). 1998 Oct;139(3): 239-42.

48 Geyer MA, Dulawa SC. Assessment of murine startle reactivity, prepulse inhibition, and habituation. Curr Protoc Neurosci. 2003 Nov;Chapter 8:Unit 8.17.

49 Mills KL, Bathula D, Dias TG, Iyer SP, Fenesy MC, Musser ED, et al. Altered cortico-striatal-thalamic connectivity in relation to spatial working memory capacity in children with ADHD. Front Psychiatry. 2012 Jan;3:2.

50 Li B, Mody M. Cortico-Striato-Thalamo-Cortical Circuitry, Working Memory, and Obsessive-Compulsive Disorder. Front Psychiatry. 2016 May;7:78.

51 Avram M, Brandl F, Bäuml J, Sorg C. Corticothalamic hypo- and hyperconnectivity extend consistently to basal ganglia in schizophrenia. Neuropsychopharmacology. 2018 Oct; 43(11):2239-48. 